\title{
Fresnel Region Fields of Circular Aperture Antennas
}

\author{
Ming-Kuei $\mathrm{Hu}$
}

(July 5, 1960; revised July 29, 1960)

\begin{abstract}
A different approach to the Fresnel region field approximation is introduced. Instead of using the conventional truncated power series expansion approximation, the Newton's iteration formula for square root is used. By using such an approximation for circular apertures with tapered illumination of the form $\left(1-\zeta^{2}\right)^{n}$, the Fresnel region fields can finally be expressed in terms of a new class of functions $W_{0}^{n}(\gamma, u)$. The function $W_{0}^{0}$ is shown to be related to the Lommel's functions of two variables, and the function $W_{0}^{n}$ is then obtained from the function $W_{0}^{n-1}$ by a simple recurrence relationship. Field distributions for $n=0,1,2,3,4$ and at distances $1 / 4 D^{2} / \lambda, 1 / 3 D^{2} \lambda, 1 / 2 D^{2} / \lambda, D^{2} / \lambda, 2 D^{2} / \lambda$, $\infty$ have been computed and presented as sets of curves. General and quantitative properties of the fields are clearly demonstrated by these curves. It is also shown that the field of any other nonuniform illumination with circular symmetry can be expressed in terms of the fields of the basic illumination of the form $\left(1-\zeta^{2}\right)^{n}$.
\end{abstract}

\section{Introduction}

It is well known that there is a close similarity between diffraction problems in optics and aperture field problems in antenna theory. In optics, two diffraction problems - the diffraction from a single edge or a slit and the diffraction from a circular aperture-have been well treated. These two problems were first solved by Fresnel and Lommel, respectively, in terms of the now so-called Fresnel integrals and Lommel's functions of two variables. The corresponding problems in antenna theory are the Fresnel region fields produced by rectangular and circular apertures. However, in optics, the primary illumination is assumed to be uniform over the aperture, but in antenna theory, the case of nonuniform illumination is of great interest.

For the case of uniform illumination, the analytical methods developed in optics can also be applied to antenna problems. For Fresnel region fields of rectangular apertures, generalizations to include nonuniform illumination through expansions in terms of different basic forms are not too difficult, polynomial and trigonometric sum types of illumination were treated by the author [1]. ${ }^{2}$ On the other hand, the analysis of Fresnel region fields of circular aperture antennas with nonuniform illumination is considerably more difficult. However, with the use of illumination of the form $\left(1-\zeta^{2}\right)^{n}$, the Fresnel region field was solved by the present author [1]. A short communication about this result has been published [2]. The complete analysis and numerical results are presented in this paper. A refined but more complicated result for the case of uniform illumination was given by Hansen and Bailin [3]. It may be noted that the corresponding far field problems have been treated by many authors with the use of different basic forms $[4,5,6,7]$, including the form $\left(1-\zeta^{2}\right)^{n}[4,5]$ used in this paper.

\section{Fresnel Region Approximation}

In the study of field distributions of linearly polarized uniform phase plane aperture antennas [8] the following formula [1] or a slightly different form given by Silver [8] may be used as a starting point.

$$
E(x, y, z)=\frac{j k}{4 \pi} \int_{A} F(\xi, \eta) \frac{e^{-j k r_{0}}}{r_{0}}\left(1+\frac{z}{r_{0}}\right) d A
$$

${ }^{1}$ Contribution from Department of Electrical Engineering, Syracuse University, Syracuse 10, N.Y. This work is part of a project sponsored by Rome Air Development Center under Contract No. AF 30(602)-928 with Syracuse Univ.

${ }^{2}$ Figures in brackets indicate the literature references at the end of this paper. 
where

$E(x, y, z)=$ the complex magnitude (amplitude and phase) of the electric field intensity at the field point $(x, y, z)$,

$F(\xi, \eta)=$ the illumination at the point $(\xi, \eta, 0)$ in the apperture $A$. For uniform phase illumination, $F(\xi, \eta)$ can be taken as a real function of $\xi$ and $\eta$,

$r_{0}=\sqrt{(x-\xi)^{2}+(y-\eta)^{2}+z^{2}}=$ the distance between the field point $(x, y, z)$ and the source point $(\xi, \eta, 0)$, and

$k=\frac{2 \pi}{\lambda} \quad$ with $\lambda=$ the wavelength.

The coordinate system used for writing formula (1) is given in figure 1. Formula (1) holds for any illumination function $F(\xi, \eta)$ and for apertures of any shape. In the present paper, it is assumed that the aperture is circular and the illumination is of circular symmetry.

For a circular aperture of radius $a$, as given in figure 2, formula (1) can be rewritten as follows:

where

$$
E(r, \theta, \phi)=\frac{j k}{4 \pi} \int_{0}^{2 \pi} \int_{0}^{a} F\left(\rho, \phi^{\prime}\right) \frac{e^{-j k r_{0}}}{r_{0}}\left(1+\frac{r}{r_{0}} \cos \theta\right) \rho d \rho d \phi^{\prime}
$$

$$
r_{0}=\sqrt{\left(r \sin \theta \cos \phi-\rho \cos \phi^{\prime}\right)^{2}+\left(r \sin \theta \sin \phi-\rho \sin \phi^{\prime}\right)^{2}+(r \cos \theta)^{2}} .
$$

The evaluation of the above integral is a very difficult one without further approximation. In order to facilitate its evaluation, different approximations are generally used. Depending upon the approximation used, the result may be classified as the far field approximation, the Fresnel region approximation, etc. The most widely used approach to such approximations is to expand the distance $r_{0}$, appearing in the exponential, into a power series involving $r$, and $\phi$. The result obtained, by neglecting all terms of order higher than one or of order higher than two, is generally considered as the far field approximation or the Fresnel region approximation. As far as the remaining $r$ 's under the integral sign are concerned, much more crude approximations are satisfactory. If the field point in consideration is not too close to the aperture and $\theta$ is not too large, then the factor $1 / r_{0}\left(1+\left(r / r_{0}\right) \cos \theta\right)$ can be approximated to good accuracy by using the relation,

$$
\frac{1}{r_{0}}\left(1+\frac{r}{r_{0}} \cos \theta\right) \cong \frac{2}{r}
$$

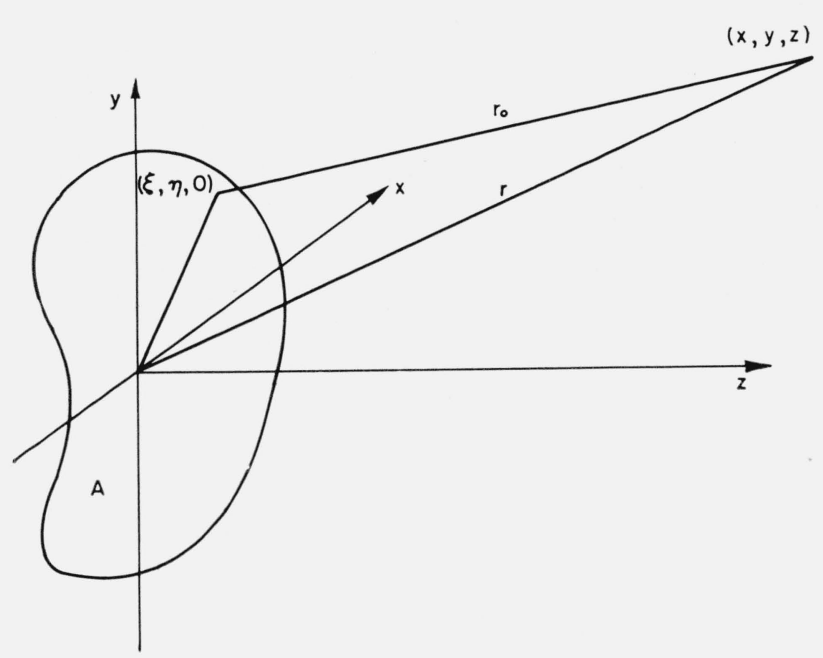

Figure 1. Coordinate system for A plane aperture.

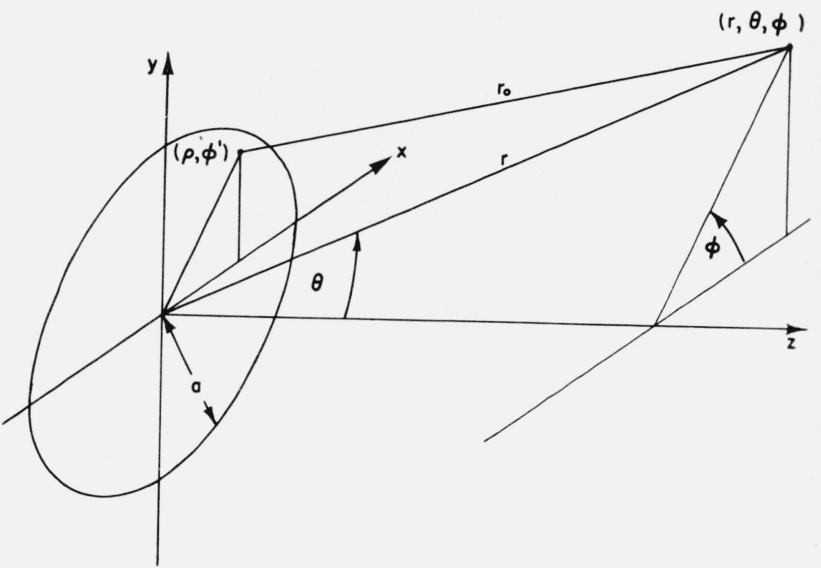

F1GURE 2. Coordinate system for A circular aperture. 
A rather different approach to the Fresnel region approximation is used in the present paper. Instead of using the truncated power series expansion, the well-known Newton's iteration formula for finding square root of a given number is employed. This formula has the following special property. If an approximation $B_{1}$, of $\sqrt{A}$ is known to be correct to $n$ significant figures, then the approximation $B_{2}$ of $\sqrt{A}$ obtained by using the iteration formula,

$$
B_{2}=\frac{1}{2}\left(B_{1}+\frac{A}{B_{1}}\right)
$$

will be correct to $2 n$ significant figures. If this formula is applied to the approximation of $r_{0}$ in Fresnel region, we have

$$
r_{0} \cong \frac{1}{2}\left\{r+\frac{\left(r \sin \theta \cos \phi-\rho \cos \phi^{\prime}\right)^{2}+\left(r \sin \theta \sin \phi-\rho \sin \phi^{\prime}\right)^{2}+(r \cos \theta)^{2}}{r}\right\} .
$$

After simplifying, eq (5) becomes

$$
r_{0} \cong r-\rho \sin \theta \cos \left(\phi-\phi^{\prime}\right)+\frac{\rho^{2}}{2 r} .
$$

This approximation of $r_{0}$ gives a simpler form than that given by Silver [8] and also by Hansen and Bailin [3]. It should be noted that this particular form makes the following relatively simple analysis possible.

Using the approximations (3) and (6), formula (2) gives:

$$
E(r, \theta, \phi)=j k \frac{e^{-j k r}}{2 \pi r} \int_{0}^{2 \pi} \int_{0}^{a} F\left(\rho, \phi^{\prime}\right) e^{j k\left[\rho \sin \theta \cos \left(\phi-\phi^{\prime}\right)-\frac{\rho^{2}}{2 r}\right]} \rho d \rho d \phi^{\prime} .
$$

If it is further assumed that the aperture illumination $F\left(\rho, \phi^{\prime}\right)$ is of circular symmetry, i.e., $F\left(\rho, \phi^{\prime}\right)$ can be written as $F(\rho)$; then $E(r, \theta, \phi)$ will be independent of $\theta$ and can be denoted by $E(r, \theta)$. Hence we have

$$
E(r, \theta)=j k \frac{e^{-j k r}}{r} \int_{0}^{a} F(\rho) J_{0}(k \sin \theta \rho) e^{-j k\left(\frac{\rho^{2}}{2 r}\right)} \rho d \rho .
$$

If a normalized variable

$$
\zeta=\frac{\rho}{a}
$$

is defined, then eq (8) can be written as

$$
E(r, \theta)=j k a^{2}\left(\frac{e^{-j k r}}{r}\right) \int_{0}^{1} F(\zeta) J_{0}(u \zeta) e^{-j \frac{\gamma}{2} \zeta^{2}} \zeta d \zeta,
$$

where

$$
u=k a \sin \theta
$$

and

$$
\gamma=\frac{k a^{2}}{r} \text {. }
$$

From eqs (11) and (12) it is clear that $u=\gamma$ implies $r \sin \theta=a$; therefore $u=\gamma$ means that the field point is at a distance, equal to the radius of the aperture, from the aperture-axis.

It is more convenient for the following discussions to rewrite eq (10) as:

$$
E(r, \theta)=e^{-j k r}\left(\gamma e^{j \frac{\pi-\gamma}{2}}\right) \int_{0}^{1} F(\zeta) J_{0}(u \zeta) e^{j \frac{\gamma}{2}\left(1-\zeta^{2}\right)} \zeta d \zeta .
$$




\section{Uniform Aperture Illumination}

For the case of uniform illumination, $F(\zeta)=1$, eq (13) reduces to

$$
E(r, \theta)=e^{-j k r}\left(\gamma e^{j \frac{\pi-\gamma}{2}}\right) \int_{0}^{1} J_{0}(u \zeta) e^{j \frac{\gamma}{2}\left(1-\zeta^{2}\right)} \cdot \zeta d \zeta
$$

In the above equation, the integral may be denoted by $W_{0}^{0}(\gamma, u)$,

$$
W_{0}^{0}(\gamma, u)=\int_{0}^{1} J_{0}(u \zeta) e^{j \frac{\gamma}{2}\left(1-\zeta^{2}\right)} \zeta d \zeta .
$$

This integral has an imaginary part as well as a real part. It is closely related to the Lommel's functions of two variables [9]. The Lommel's functions of two variables can be expressed in integral forms as:

$$
\begin{aligned}
& U_{1}(\gamma, u)=\gamma \int_{0}^{1} J_{0}(u \zeta) \cos \frac{\gamma}{2}\left(1-\zeta^{2}\right) \cdot \zeta d \zeta, \\
& U_{2}(\gamma, u)=\gamma \int_{0}^{1} J_{0}(u \zeta) \sin \frac{\gamma}{2}\left(1-\zeta^{2}\right) \cdot \zeta d \zeta .
\end{aligned}
$$

$U_{1}(\gamma, u)$ and $U_{2}(\gamma, u)$ are of order one and order two, respectively.

By integrating by parts and using the recurrence relations of Bessel functions, it can be proved that $U_{1}(\gamma, u)$ and $U_{2}(\gamma, u)$ may be expanded into the following two series:

$$
\begin{aligned}
& U_{1}(\gamma, u)=\left(\frac{\gamma}{u}\right) J_{1}(u)-\left(\frac{\gamma}{u}\right)^{3} J_{3}(u)+\left(\frac{\gamma}{u}\right)^{5} J_{5}(u) \ldots \\
& U_{2}(\gamma, u)=\left(\frac{\gamma}{u}\right)^{2} J_{2}(u)-\left(\frac{\gamma}{u}\right)^{4} J_{4}(u)+\left(\frac{\gamma}{u}\right)^{6} J_{6}(u) \ldots
\end{aligned}
$$

Generalizations of these functions to higher orders are possible, but these generalizations will not be used in the present paper.

In terms of $U_{1}(\gamma, u)$ and $U_{2}(\gamma, u)$, we have

$$
W_{0}^{0}(\gamma, u)=\frac{U_{1}(\gamma, u)}{\gamma}+j \frac{U_{2}(\gamma, u)}{\gamma} .
$$

Using the above relation for $W_{0}^{0}(\gamma, u)$, formula (14) can be written simply as:

$$
E(r, \theta)=e^{-j k r}\left(\gamma e^{j \frac{\pi-\gamma}{2}}\right) \cdot W_{0}^{0}(\gamma, u) .
$$

This result is essentially the same as the first term in the series solution of Hansen and Bailin [3]. It can be seen from their figure 6 that the two results are close to each other within the region considered in this paper.

\section{Nonuniform Aperture Illumination of the Form $\left(1-\zeta^{2}\right)^{n}$}

If the aperture illumination is assumed to be of the form $\left(1-\zeta^{2}\right)^{n}$, the field expression can be obtained by substituting $F(\zeta)=\left(1-\zeta^{2}\right)^{n}$ into eq (13), that gives

$$
E(r, \theta)=e^{-j k r}\left(\gamma e^{j \frac{\pi-\gamma}{2}}\right) \int_{0}^{1}\left(1-\zeta^{2}\right)^{n} J_{0}(u \zeta) e^{j \frac{\gamma}{2}\left(1-\zeta^{2}\right)} \zeta d \zeta .
$$


Now if we define

$$
W_{0}^{n}(\gamma, u)=\int_{0}^{1}\left(1-\zeta^{2}\right)^{n} J_{0}(u \zeta) e^{j \frac{\gamma}{2}\left(1-{ }^{2}\right)} \zeta d \zeta
$$

then

$$
E(r, \theta)=e^{-j k r}\left(\gamma e^{j \frac{\pi-\gamma}{2}}\right) \cdot W_{0}^{n}(\gamma, u)
$$

When $n=0$, eqs (20) and (22) reduce to eqs (14) and (19), respectively. In other words eqs (20) and (22) include uniform illumination as a special case. For $n \neq 0$, these give the field distributions for the special tapered illumination $\left(1-\zeta^{2}\right)^{n}$. The integration of the integral (21), or the evaluation of the function $W_{0}^{n}(\gamma, u)$, can be carried out by noting the following simple property: If differentiation of $W_{0}^{n}(\gamma, u)$ with respect to $\gamma$ is carried out, we have

$$
\frac{\partial}{\partial \gamma} W_{0}^{n}(\gamma, u)=\frac{j}{2} \int_{0}^{1}\left(1-\zeta^{2}\right)^{n+1} J_{0}(u \zeta) e^{j \frac{\gamma}{2}\left(1-\zeta^{2}\right)} \zeta d \zeta .
$$

Equation (23) gives the recurrence relation,

$$
W_{0}^{n+1}(\gamma, u)=\frac{2}{j} \frac{\partial}{\partial \gamma} W_{0}^{n}(\gamma, u) .
$$

In terms of $W_{0}^{0}(\gamma, u)$, we have

or

$$
W_{0}^{n}(\gamma, u)=\left(\frac{2}{j}\right)^{n} \frac{\partial^{n}}{\partial \gamma^{n}} W_{0}^{0}(\gamma, u)
$$

$$
W_{0}^{n}(\gamma, u)=\left(\frac{2}{j}\right)^{n}\left[\frac{\partial^{n}}{\partial \gamma^{n}} \frac{U_{1}(\gamma, u)}{\gamma}+j \frac{\partial^{n}}{\partial \gamma^{n}} \frac{U_{2}(\gamma, u)}{\gamma}\right] .
$$

It is possible to derive expressions of $W_{0}^{n}(\gamma, u)$ in terms of Lommel's functions of orders higher than two. But as far as numerical evaluation is concerned, it was found that the simplest way is to use eq (26) and the series expansions (17) for $U_{1}(\gamma, u)$ and $U_{2}(\gamma, u)$. The series expansions of $\left(\partial^{n} / \partial \gamma^{n}\right)\left(U_{1}(\gamma, u) / \gamma\right)$ and $\left(\partial^{n} / \partial \gamma^{n}\right)\left(U_{2}(\gamma, u) / \gamma\right)$, for $n=0,1,2,3$, and 4 , are obtained by differentiating the power series of $U_{1}(\gamma, u) / \gamma$ and $U_{2}(\gamma, u) / \gamma$, term by term, $n$ times with respect to $\gamma$.

\section{Results}

In the last section, for the aperture illumination of the form $\left(1-\zeta^{2}\right)^{n}$, the field expression is given by

where

$$
E(r, \theta)=e^{-j k r}\left(\gamma e^{j \frac{\pi-\gamma}{2}}\right) \cdot W_{0}^{n}(\gamma, u),
$$

$$
\gamma=\frac{k a^{2}}{r}
$$

and

$$
u=k a \sin \theta .
$$

$E(r, \theta)$ in eq $(27)$ can always be rewritten in the following form:

$$
E(r, \theta)=A e^{j 2 \pi \phi}
$$

where $A$ is the amplitude and $2 \pi \phi$ is the phase angle in radians.

Plots of the aperture illumination $\left(1-\xi^{2}\right)^{n}$ for $n=0,1,2,3,4$, are given in figure 3 . The field distribution curves, both amplitude $A$ and phase $2 \pi \phi$, are computed for the following cases (see fig. 4): 


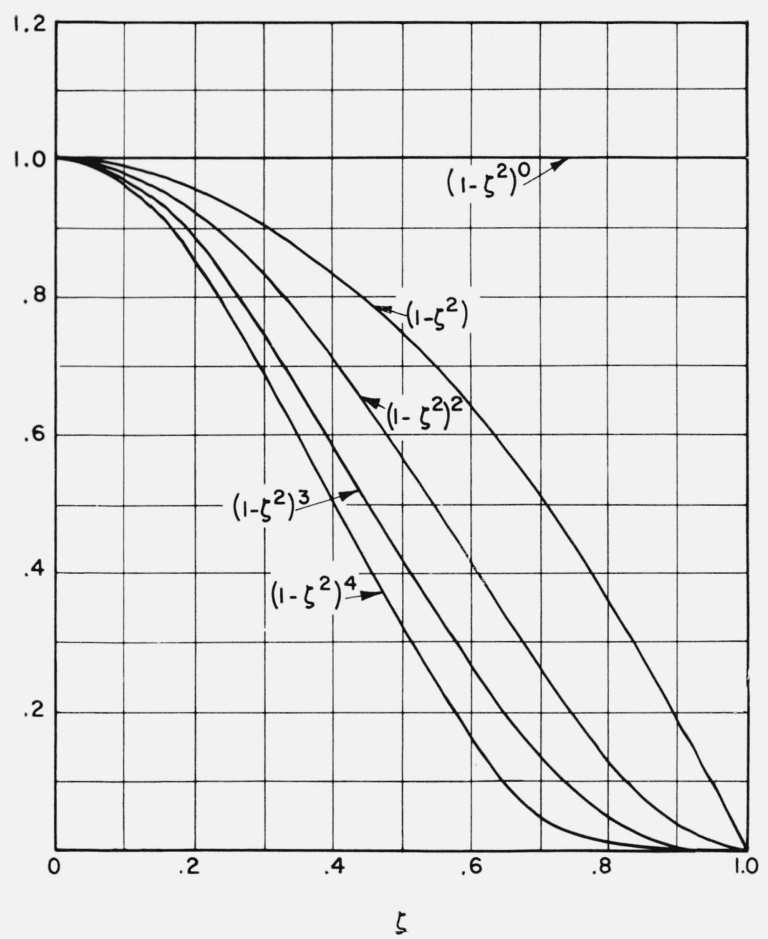

Figure 3. Aperture illumination distributions, uniform and tapered.

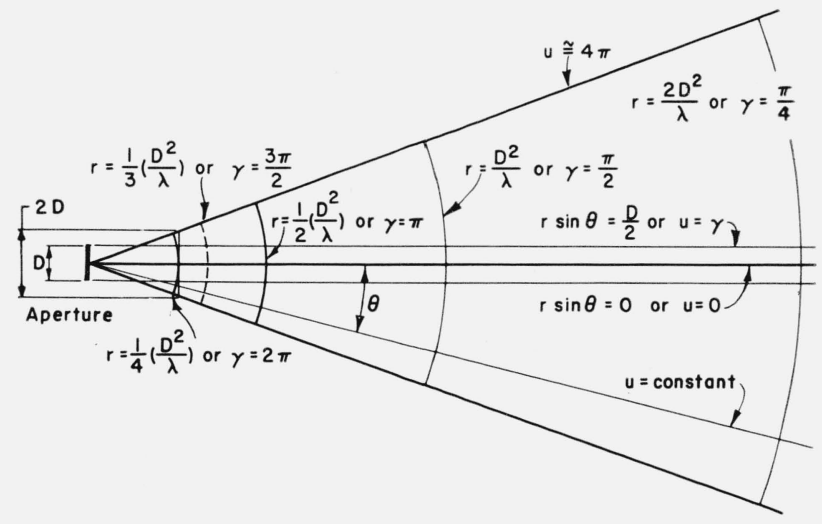

Figure 4. Fresnel region, in which field distributions have been computed.

(i) Field distributions along the circular ares:

$$
r=\frac{1}{4}\left(\frac{D^{2}}{\lambda}\right), \frac{1}{3}\left(\frac{D^{2}}{\lambda}\right), \frac{1}{2}\left(\frac{D^{2}}{\lambda}\right),\left(\frac{D^{2}}{\lambda}\right), 2\left(\frac{D^{2}}{\lambda}\right), \infty,
$$

where $D=2 a$. The corresponding values for $\gamma$ are:

$$
\gamma=2 \pi, \frac{3 \pi}{2}, \pi, \frac{\pi}{2}, \frac{\pi}{4}, 0 .
$$

It should be noted that the case $r=\infty$ or $\gamma=0$ is the conventional far field result.

For the convenience of comparison, the field distribution curves are normalized in the following way: Let

$$
E(r, 0)=A_{0} e^{j 2 \pi \phi_{0}}
$$

then the normalized $E(r, \theta)$ for a fixed $r$ is given by

$$
\frac{E(r, \theta)}{E(r, 0)}=\frac{A}{A_{0}} e^{j 2 \pi\left(\phi-\phi_{0}\right)}=A_{n} e^{j 2 \pi \phi_{n}} .
$$

The normalized amplitude and phase distributions along the ares are given in figure 5 to figure 14 .

(ii) Field distributions along the aperture-axis:

In this and the following case, the distributions are not normalized but are plotted with the factor $e^{-j k r}$ excluded. The two distribution curves, amplitude and phase, in the present case are useful in correlating the normalized distributions given in case (i).

(iii) Field distributions along a line parallel to the aperture-axis and at a distance equal to the radius of the aperture: 


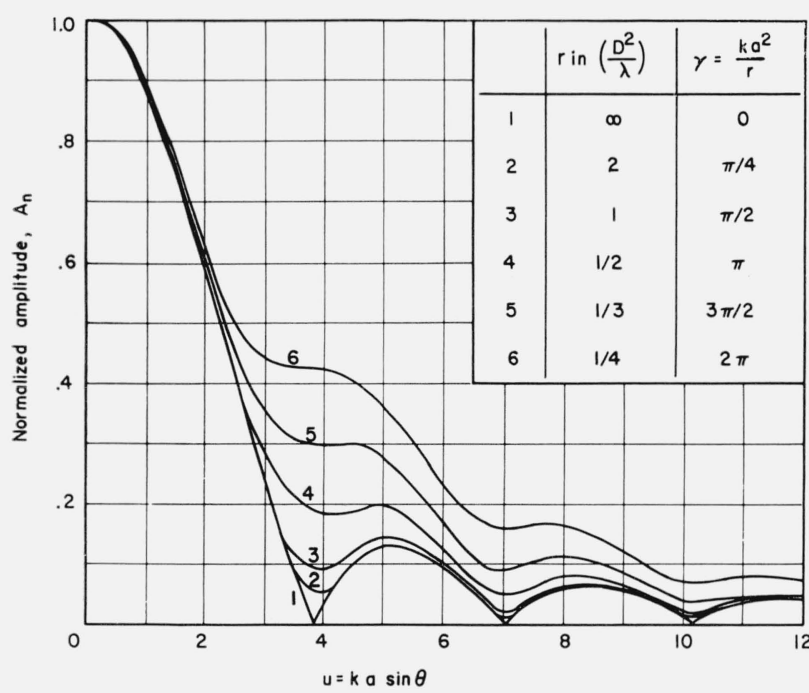

FIGURE 5. Normalized amplitude distribution curves with aperture illumination $=\left(1-\zeta^{2}\right)^{0}$, (constant).

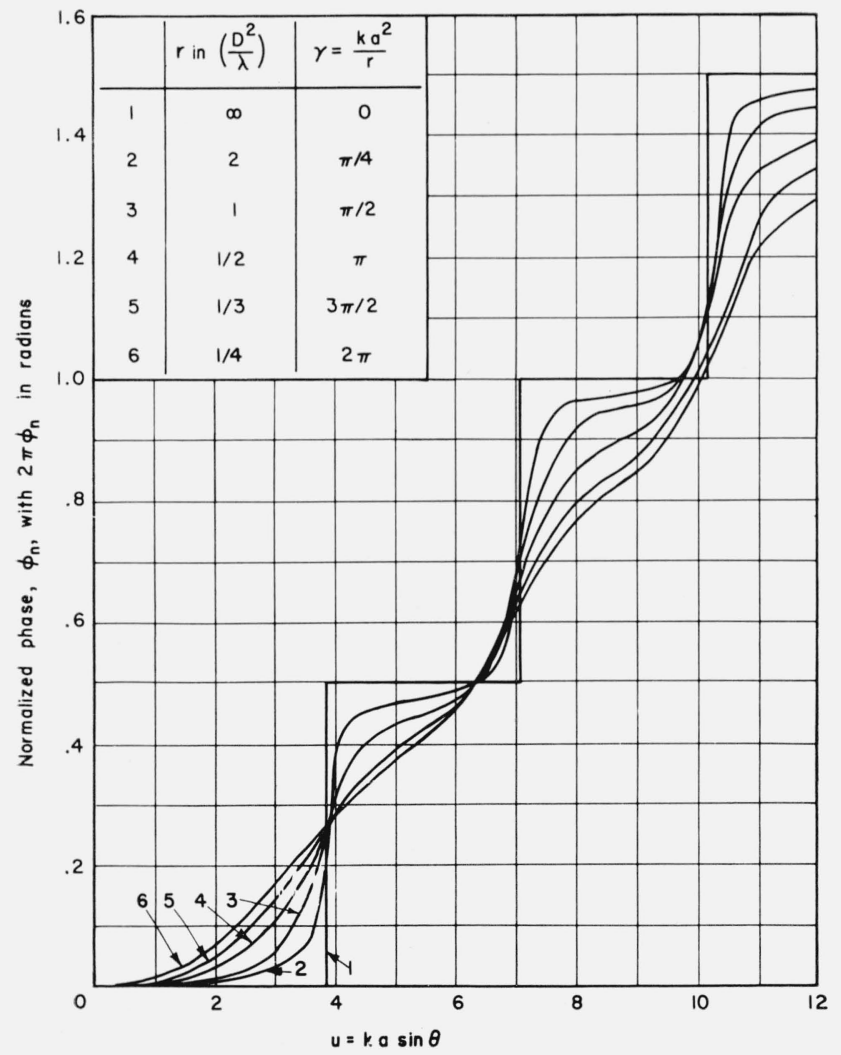

Figure 6. Normalized phase distribution curves with aperture illumination $=\left(1-\zeta^{2}\right)^{0}$, (constant).

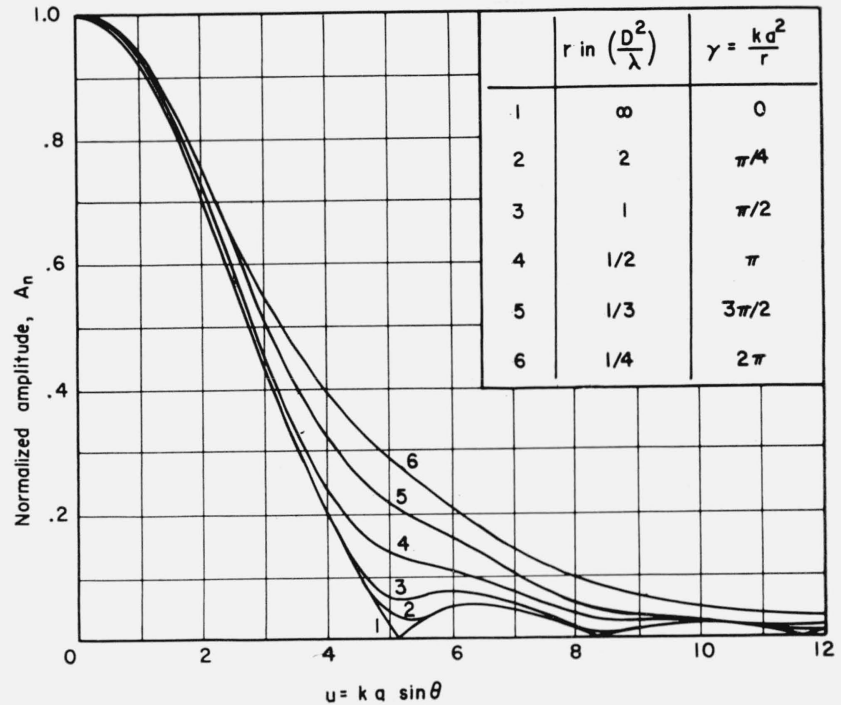

Figure 7. Normalized amplitude distribution curves with aperture illumination $=\left(1-\zeta^{2}\right)$.

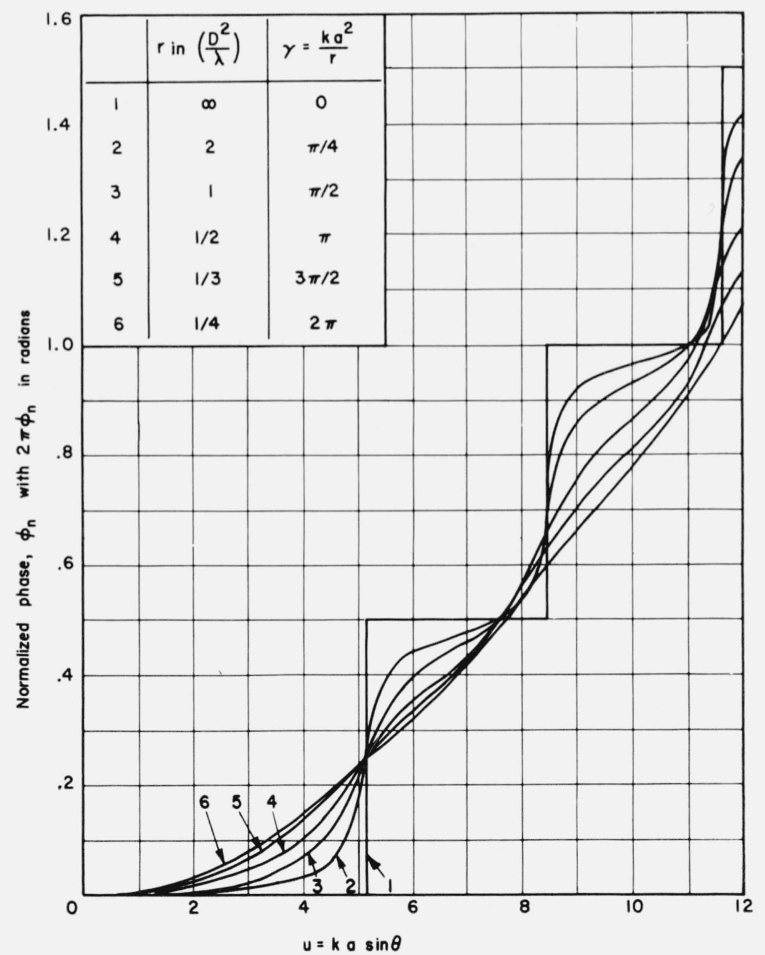

FIGURE 8. Normalized phase distribution curves with aperture illumination $=\left(1-\zeta^{2}\right)$. 


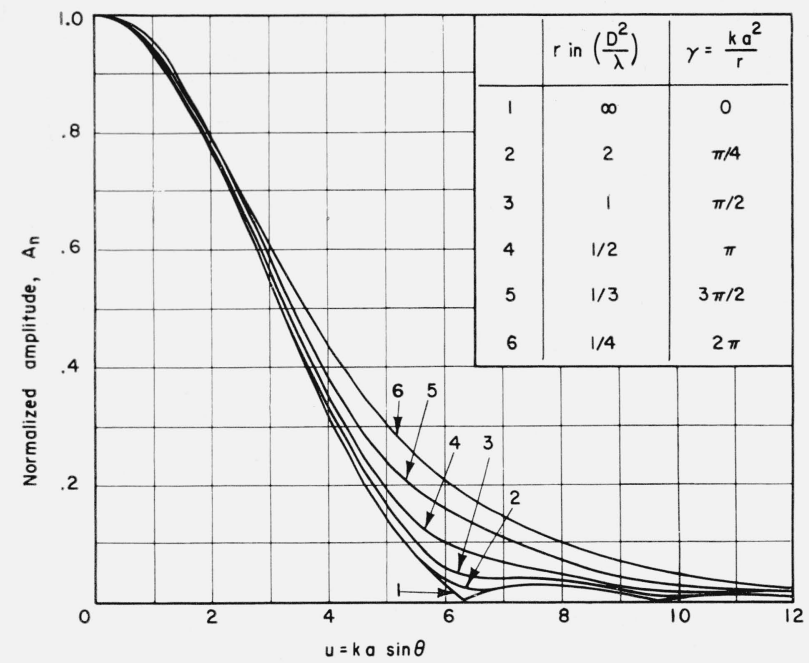

FIgure 9. Normalized amplitude distribution curves with aperture illumination $=\left(1-\zeta^{2}\right)^{2}$.

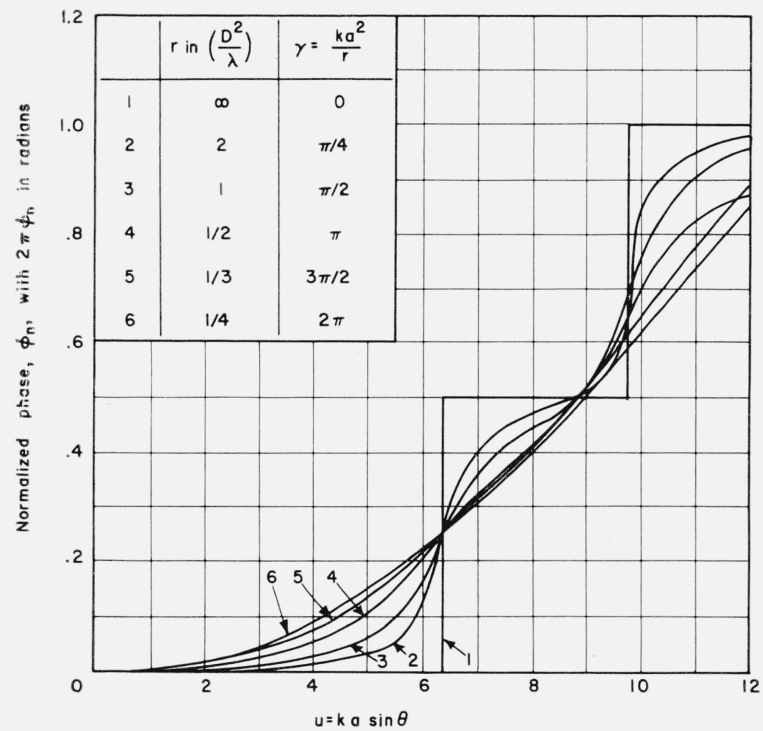

FIGURE 10. Normalized phase distribution curves with aperture illumination $=\left(1-\zeta^{2}\right)^{2}$.

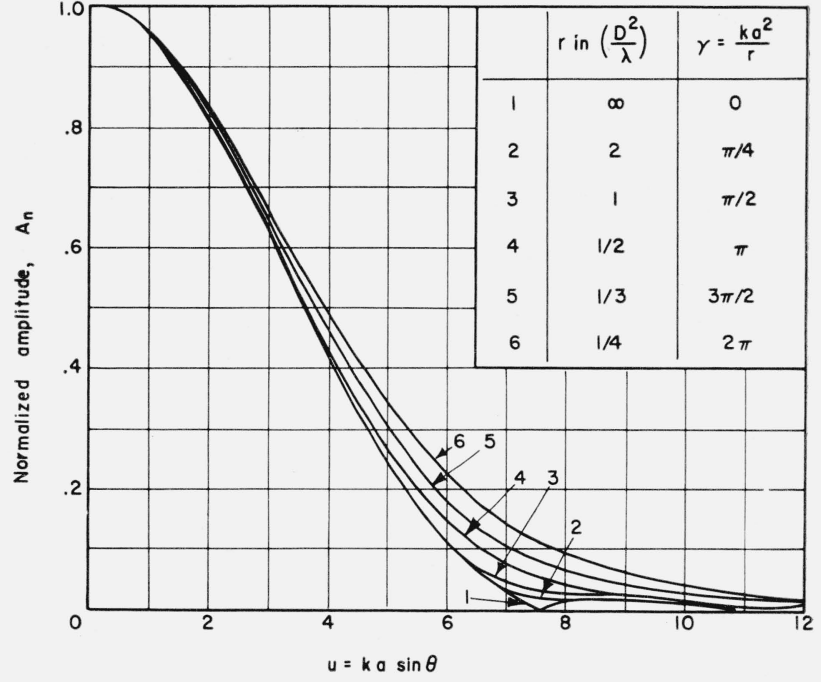

Figure 11. Normalized amplitude distribution curves with aperture illumination $=\left(1-\zeta^{2}\right)^{3}$.

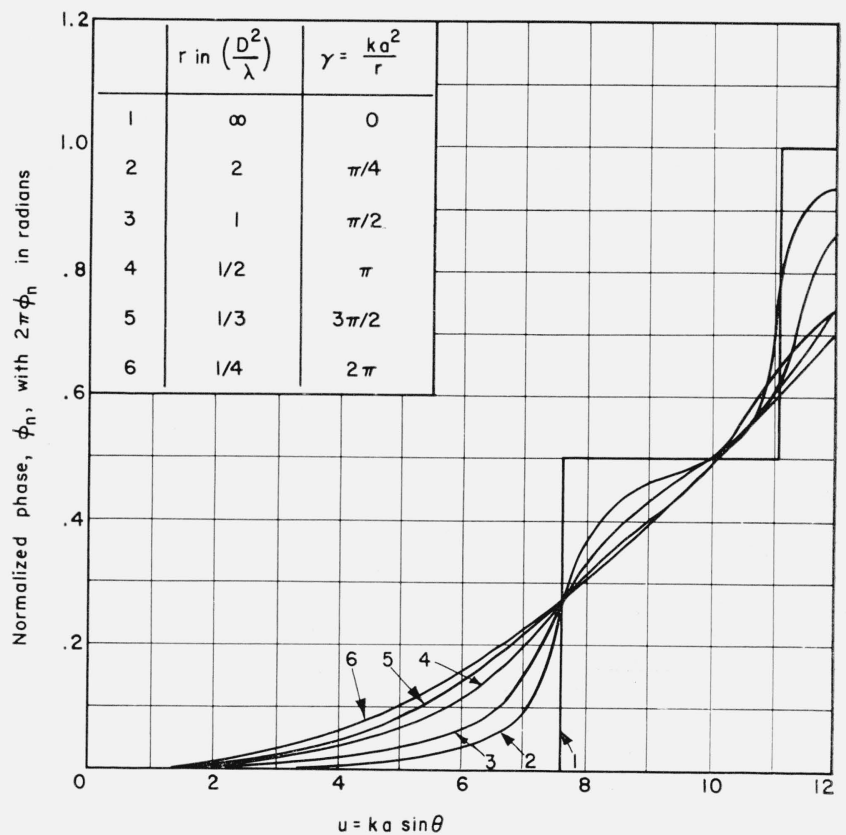

FIGURE 12. Normalized phase distribution curves with aperture illumination $=\left(1-\xi^{2}\right)^{3}$.

Distribution curves for case (ii) and cases (iii) are given in figure 15 to figure 18 . From these field distribution curves, the following properties are observed.

(1) For the normalized field distribution curves along circular arcs:

(a) For each aperture illumination of the form $\left(1-\zeta^{2}\right)^{n}$, the main beam width is essentially a constant in the range of $r$ from $\frac{1}{8}\left(2 D^{2} / \lambda\right)$ up to infinity. (Note: For a fixed direction, the parameter $u$ is a constant.)

(b) There is no perceptible difference in the normalized amplitude distribution curves at $2 D^{2} / \lambda$ (conventional far field limit) and at infinity for the same aperture illumination, except near those directions where the infinity case has zero values or nulls. On the other hand, there are differences between the phase distribution curves; these curves change from a stepped form at infinity to a smooth continuous form at $2 D^{2} / \lambda$. 


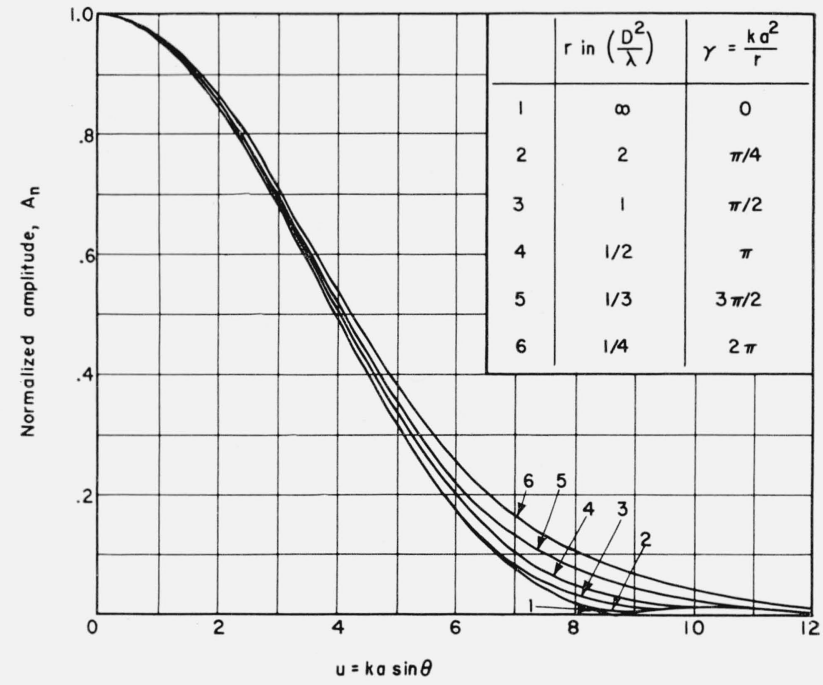

Figure 13. Normalized amplitude distribution curves with aperture illumination $=\left(1-\zeta^{2}\right)^{4}$.

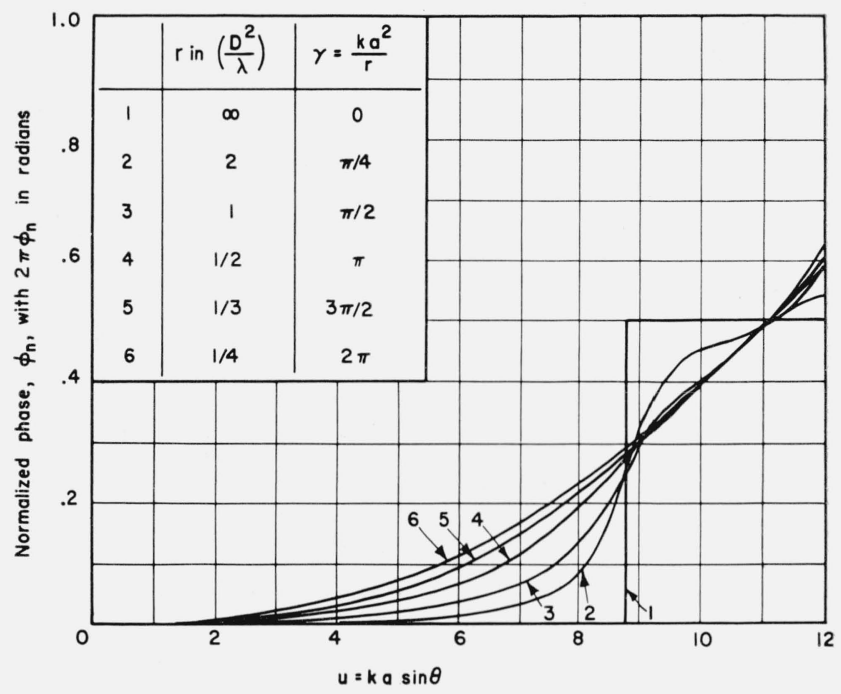

FIGURE 14. Normalized phase distribution curves with aperture illumination $=\left(1-\zeta^{2}\right)^{4}$.

(c) The "side lobe level" increases as the distance $r$ is reduced, but the amplitude distribution curve as a whole becomes "smoother." It is also true that the phase distribution curve beromes smoother too as $r$ is reduced.

(d) When the distribution curves of different aperture illuminations are compared, it is seen that the main lobe beamwidth increases as $n$ is increased. At the same time, within a fixed measure of the angle $\theta$, the number of side lobes is reduced.

(e) The side lobe level is definitely reduced as $n$ is increased. The ratio of the normalized maximum amplitudes of the first side lobes with $n=4$ and $n=0$ is approximately 1 to 10 .

(f) As $n$ increases, the difference in the normalized amplitude distribution curves at different $r$ becomes smaller.

(2) For the field distribution curves along the aperture-axis:

(a) Along the aperture-axis, the amplitude distributions vary closely as $1 / r$ in the range $r=\frac{1}{2}\left(2 D^{2} / \lambda\right)$ to $r=$ infinity for all cases studied. As for the particular case $n=4$, this is true even when $r$ is reduced to $1 / 8\left(2 D^{2} / \lambda\right)$.

(b) Along the aperture-axis, all the phase distributions, excluding the exponential factor $e^{-j k r}$, are approximately straight lines, i.e., the phase varies linearly with respect to $1 / r$.

(3) For the field distribution curves along a line parallel to the aperture-axis and at a distance equal to the radius of the aperture;

(a) In the range, $r=2 D^{2} / \lambda$ to $r=$ infinity, both the amplitude and phase distributions have approximately the same values as those obtained along the aperture-axis.

(b) At $r=1 / 8\left(2 D^{2} / \lambda\right)$, the ratio of the corresponding amplitudes for the present case and case

(ii) is roughly one to four for all cases computed.

\section{Generalization to Nonuniform Aperture Illuminations of Other Forms}

In the last section, only a special type of nonuniform illumination of the form $\left(1-\zeta^{2}\right)^{n}$ was given. It is possible to generalize the analysis to include nonuniform illuminations of other forms either through expansions by the Schmidt orthogonalization process [5] or by the following simple algebraic procedure. For any circularly symmetric aperture illumination, it is always possible to approximate it by an even polynomial of the variable $\zeta$. Such poly- 


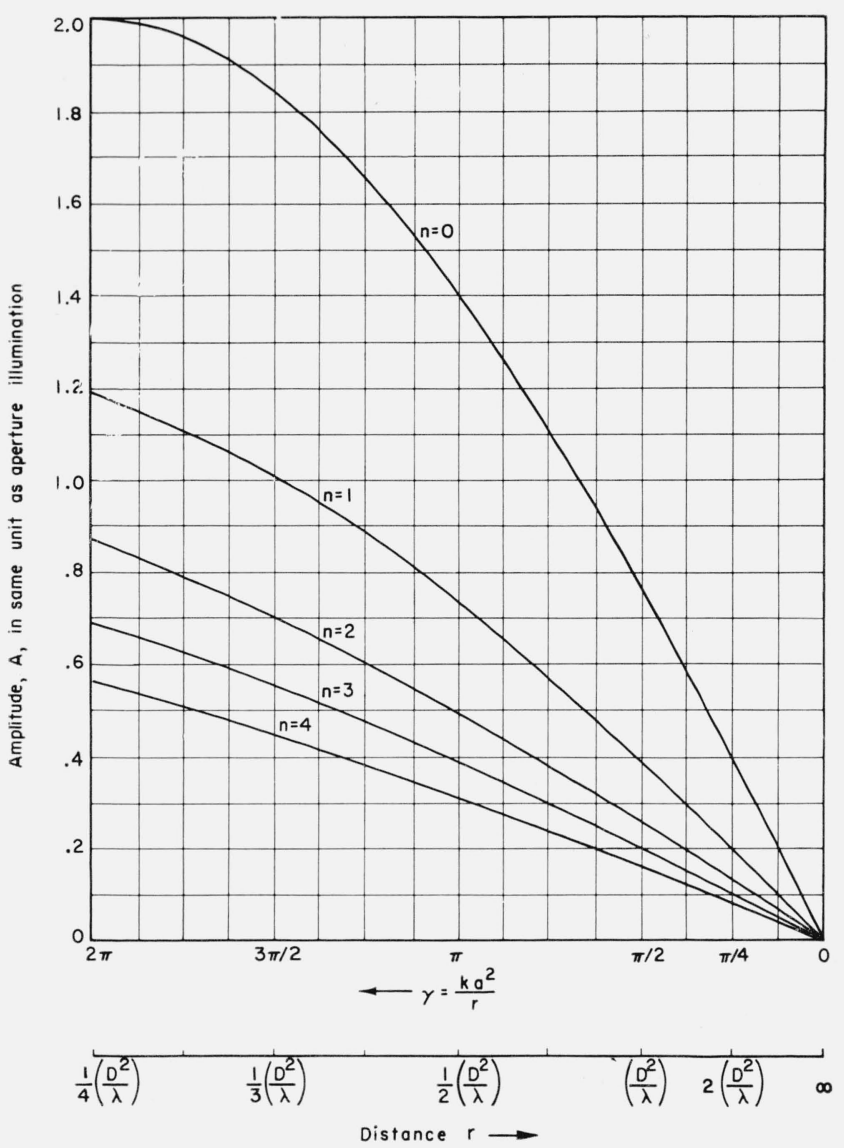

Figure 15. Amplitude distribution curves along the aperture axis with aperture illumination $=\left(1-\zeta^{2}\right)^{n}$.

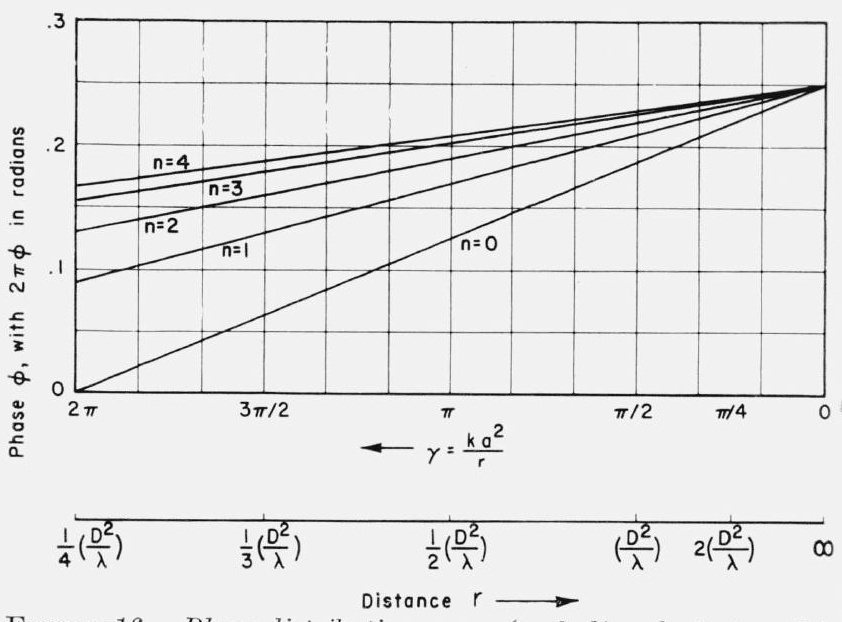

Figure 16. Phase distribution curves (excluding the factor $\mathrm{e}^{-\mathrm{j} \mathbf{k r}}$ ) along the aperture axis with aperture illumination $=\left(1-\zeta^{2}\right)^{n}$.

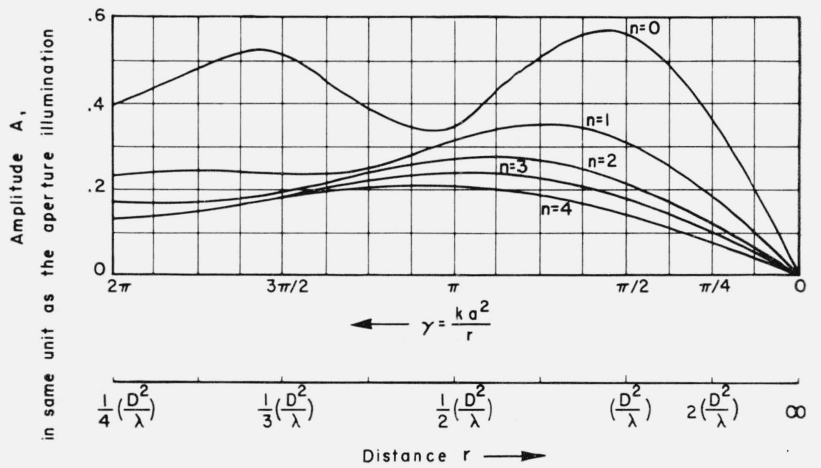

FIGURE 17. Amplitude distribution curves along a line parallel to the aperture axis and at a distance a from the aperture axis with aperture illumination $=\left(1-\zeta^{2}\right)^{n}$.

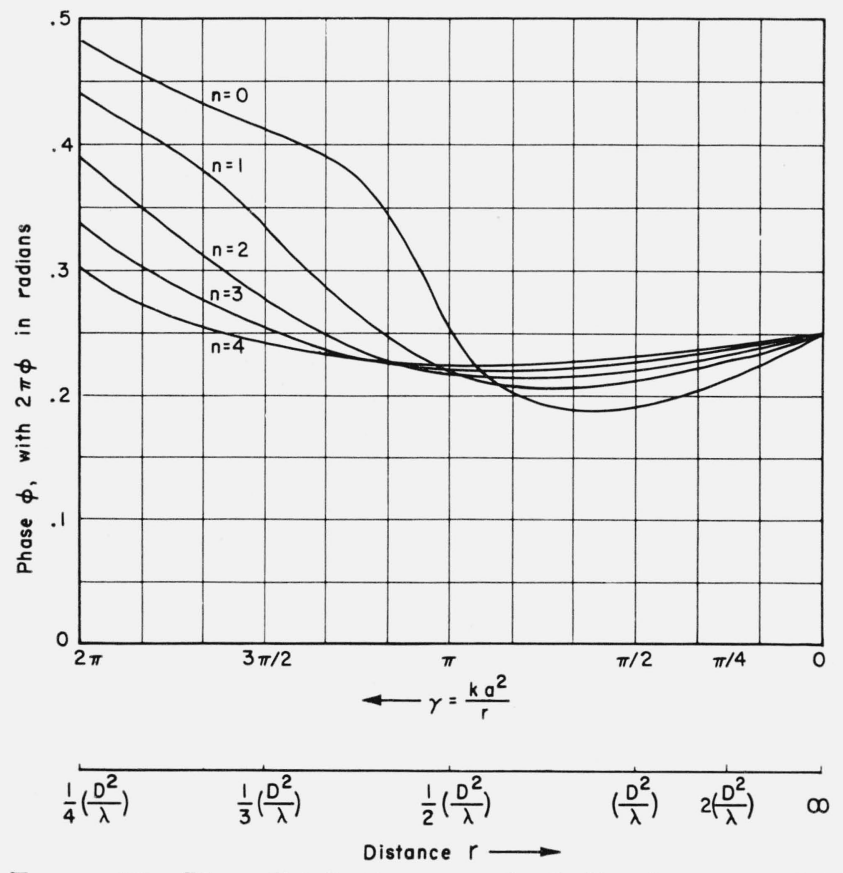

Figure 18. Phase distribution curves (excluding the factor $\mathrm{e}^{-\mathrm{jkr}}$ ) along a line parallel to and at a distance a from the aperture axis with aperture illumination $=\left(1-\zeta^{2}\right)^{n}$. 
nomials are of the following form:

$$
a_{n} \zeta^{2 n}+a_{n-1} \zeta^{2(n-1)}+\ldots+a_{1} \zeta^{2}+a_{0} .
$$

It is clear that such a polynomial can always be transformed into the following form:

$$
b_{n}\left(1-\zeta^{2}\right)^{n}+b_{n-1}\left(1-\zeta^{2}\right)^{n-1}+\ldots+b_{1}\left(1-\zeta^{2}\right)+b_{0} .
$$

For a given set of $a$ 's, the corresponding $b$ 's can be uniquely determined by solving a system of linear simultaneous algebraic equations. In particular, for the case of $n=4$, the coefficients $b$ 's obtained in terms of the $a$ 's are as follows:

$$
\begin{aligned}
& b_{4}=a_{4} \\
& b_{3}=-a_{3}-4 a_{4} \\
& b_{2}=a_{2}+3 a_{3}+6 a_{4} \\
& b_{1}=-a_{1}-2 a_{2}-3 a_{3}-4 a_{4} \\
& b_{0}=a_{0}+a_{1}+a_{2}+a_{3}+a_{4} .
\end{aligned}
$$

When $b_{0}, b_{1}, \ldots, b_{4}$ are determined as above, the field expression is then given by

$$
E(r, \theta)=e^{-j k r}\left(\gamma e^{j \frac{\pi-\gamma}{2}}\right) \int_{0}^{1} \sum_{n=0}^{4} b_{n}\left(1-\zeta^{2}\right)^{n} J_{0}(u \zeta) e^{j \frac{\gamma}{2}\left(1-\zeta^{2}\right)} \zeta d \zeta
$$

In terms of $W_{0}^{n}(\lambda, u)$, (36) gives

$$
E(r, \theta)=e^{-j k r}\left(\gamma e^{j \frac{\pi-\gamma}{2}}\right) \sum_{n=0}^{4} b_{n} W_{0}^{n}(\gamma, u)
$$

Equation (37) can then be evaluated by using the results already obtained in this paper.

\section{References}

[1] M. K. Hu, Study of near-zone fields of large aperture antennas, Syracuse Univ. Research Inst., Syracuse, New York, final rept., pt. 2 (Apr. 1957).

[2] M. K. Hu, Fresnel region field distribution of circular aperture antennas, IRE Trans. on Antennas and Propagation, AP-8, No. 3, pp. 344-346 (May 1960).

[3] Hansen and Bailin, A new method of near field analysis, IRE Trans. on Antennas and Propagation AP-y, special suppl., pp. S458-S467 (Dec. 1960).

[4] R. C. Spencer, Paraboloid diffraction patterns from the standpoint of physical optics, MIT, Radiation Lab. RL Rept. T-7 (Oct. 1942).

[5] C. T.A. Johnk, Synthesis of aperture antennas, Univ., Ill., Eng. Exptl. Sta., Tech. Rept. 1 (1954).

[6] A. Ishimaru and G. Held, Analysis and synthesis of radiation patterns from circular apertures, Can. J. Phys. 38, pp. 78-99 (Jan. 1960).

[7] T. T. Taylor, Design of circular apertures for narrow beamwidth and low sidelobes, IRE Trans. on Antenna and Propagation 8, No. 1, pp. 17-22 (Jan. 1960).

[8] S. Silver, Microwave antenna theory and design (McGraw-Hill Book Co., Ine., New York, N.Y., Radiation Lab. Ser 12, pp. 170-173, 1949).

[9] G. N. Watson, A treatise on the theory of Bessel functions (Cambridge Univ. Press, second ed., pp. 537-540, 1952).

(Paper 65D2-111) 